\title{
GOBERNANZA Y GESTIÓN DEL CONOCIMIENTO: LAS PATENTES, UN INSTRUMENTO DE ESTUDIO
}

\author{
Ana Romero de Pablos \\ Departamento de Ciencia, Tecnologia y Sociedad \\ Instituto de Filosofia, CSIC
}

\begin{abstract}
Patents play a significant role in innovation. In this essay they are used as tools for studying and analysing knowledge management. Most of the research done on patents is related to measuring and quantifying patents in the market. The aim of this paper is to present patents as instrumental intermediary agents between technoscientific production and its social uses. Scientists, scientific institutions, centres of technology transfer and industrial firms are all actors at play in this process.

In this occasion, CSIC (Spanish National Research Council) patens are analysed so as to check this kind of interaction between science and technology policies, and society. This analysis, together with specific case studies, will allow an evaluation of how CSIC patents contribute to technology develoment and transfer, how they reflect previous science policies, and how the new knowledge arising from patents generates new management models.
\end{abstract}

KEY WORDS Science and technology policies, science and society, knowledge and society, patents.
RESUMEN Las patentes juegan un importante papel en el proceso innovador de cualquier país. El presente estudio quiere presentar las patentes como agentes fundamentales de intermediación entre la producción tecnocientífica y el uso que de ella hace la sociedad. El hecho de que las patentes incorporen a todos los actores que forman parte de este proceso (los investigadores, las instituciones científicas, los organismos de gestión del conocimiento, las empresas, etc.) las hace fundamentales a la hora de abordar este estudio.

Partiendo de los datos en materia de patentes que genera el Consejo Superior de Investigaciones Científicas, se estudiará el uso inmediato que se hace de la tecnología patentada como ayuda necesaria para el desarrollo de I+D. Se estudiará la incidencia, que a más largo plazo, pueden tener las patentes en la industria y en las administraciones públicas a la hora de planificar actividades o diseñar de estrategias a seguir. Los estudios realizados hacen pensar en una posible relación directa entre política científica y patentes. Todo esto unido al estudio de casos concretos permitirá valorar en qué medida las patentes del CSIC han contribuido al desarrollo del estado de la técnica de un sector determinado, cómo ha sido la evolución tecnológica que se ha producido, cómo se ha generado la transferencia de tecnología, en qué medida las patentes del CSIC permiten hablar de un reflejo de políticas científicas anteriores, hasta que punto el nuevo conocimiento generado por las patentes repercute también una nueva forma de gestionarlo.

PALABRAS CLAVE Politicas de ciencia y tecnología, ciencia y sociedad, conocimiento y sociedad, patentes.

\section{Introducción}

Los cambios que se han producido en las nuevas formas de obtención del conocimiento han repercutido en la aparición de nuevos actores, nuevas demandas de aplicación de este conocimiento, nuevos procedimientos. Estos cambios también han permeado modificando las estructuras y organizaciones encargadas de gestionar este conocimiento incorporando en sus equipos de trabajo a otros actores, como científicos y politicos científicos, antes ausentes pero que ahora resultan muy útiles para establecer el necesario diálogo con los investigadores. El cambio en la producción de conocimiento también ha repercutido en la forma de cuantificar y medir la producción científica. Los curricula van, aunque de forma todavía tímida, incorporando nuevos espacios para nuevos méritos. En este nuevo contexto en el que hay que pensar en nuevas modos de participación de los distintos actores que forman parte del mismo, desde las politicas científicas hasta las nuevas formas de llegar e involucrar a la ciudadania, es donde hay que enmarcar este 
trabajo donde las patentes son un instrumento muy rico a la hora de estudiar no solo la producción sino también la gestión del conocimiento generado.

De todos es conocido que las patentes juegan un importante papel en el proceso innovador de cualquier país. Valorarlas y utilizarlas como instrumento para el estudio y análisis de la gestión del conocimiento es lo que se pretende en este artículo. Aunque es cierto que los trabajos que se han ocupado de estudiar las conexiones existentes entre el conocimiento generado en el ámbito de la investigación y su paso al mundo de la aplicación en España no son muy alentadores, también lo es que son procesos complejos necesitados de otros estudios y aproximaciones. Hay indicios de que esto puede estar cambiando.

Uno de ellos, aunque no el único, es la aparición del Consejo Superior de Investigaciones Científicas (CSIC), organismo público español que aglutina el mayor número de centros de investigación dedicados a ciencia y tecnología, en una Newsletter ( $n^{\circ}$ 06/2004) del Patent Corporation Treaty (PCT, Tratado de Cooperación en materia de Patentes). Esta lista hace públicas las empresas que más han utilizado, durante el año 2003, el sistema PCT. Este tratado, firmado por 121 países de todo el mundo, internacionaliza y al tiempo facilita ciertas fases del procedimiento de tramitación de patentes en cualquiera de los paises firmantes. Otro dato que hay que tener en cuenta es que el CSIC es el único organismo español que figura en esta relación, no aparece ninguna otra empresa pública o privada española, y lo hace ocupando el puesto 166 de un total de 221. Por delante en el ranking hay empresas y organismos públicos de investigación de nacionalidad alemana, japonesa, norteamericana, inglesa, sueca, francesa, coreana, suiza o india. También en estudios regionales sobre innovación y desarrollo tecnológico han aparecido datos que ponen de manifiesto el peso del CSIC como una de las principales entidades públicas de investigación titulares de patentes en la Comunidad de Madrid durante el periodo 1992-1999 (Buesa, 2002).

Si a lo anterior se une el papel que en general juegan estos documentos, las patentes, en el proceso innovador de cualquier pais, parece lógico y oportuno plantear que puedan ser un buen instrumento de trabajo que aporte datos interesantes que permitan ahondar en los estudios sobre sociedad civil y gobernanza de la ciencia y la tecnología, y más concretamente sobre patentes y gobierno estratégico de la ciencia y la tecnología. Partiendo de la definición de gobernanza como la puesta en práctica de formas de gobierno estratégicas para poner de relieve el valor de lo público a través de la relación entre sociedad, mercado y Estado y conseguir de ésta forma un desarrollo socialmente sostenible, y siguiendo la definición del concepto de usistema de innovación" propuesto por J. Metcalfe (1995), la patente aparece como un elemento interesante de intermediación entre la producción tecnocientífica y el uso que de ella hace la sociedad; también el documento de patente incorpora a todos los actores que han intervenido en el proceso (investigadores, instituciones científicas, organismos de gestión del conocimiento, empresas ...); y por último, no hay patente si no hay conocimiento.

Tradicionalmente los estudios sobre patentes han ocupado y ocupan a especialistas en economía del cambio técnico. Gracias a esos trabajos, sabemos que las patentes no solo permiten conocer las actividades de producción de conocimiento, sino que permiten también la puesta en el merca-

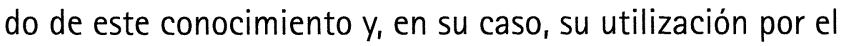
sistema productivo industrial. Los estudios disponibles sobre patentes en España se refieren en su mayoría a empresas innovadoras, aunque recientemente han comenzado a incorporar también datos significativos de universidades y de organismos públicos de investigación (sobre patentes, véase Bercovitz et al 1985; para una contextualización de los datos de patentes españolas en el marco del sistema español de I+D, véase Buesa 2003; Sanz Menéndez y Arias, 1998; Buesa y Molero 1992; Azagra, Fernández de Lucio y Gutiérrez, 2003). La mayor parte de estos análisis realizados desde una perspectiva económica persiguen conseguir un modelo econométrico que pueda ser aplicado de forma general y que permita medir y evaluar la innovación a través de las patentes. Pero la mayor parte de estos trabajos, al contextualizar los datos de las patentes en el sistema general de $I+D$, lo que hacen es medir las condiciones del entorno en el que se han generado estas patentes y hasta qué punto favorecen la innovación, o medir los factores de producción individuales o la producción en sí misma, pero los resultados obtenidos mediante estos modelos econométricos proporcionan una parte de lo ocurrido, no entrando por ejemplo en cuestiones relacionadas con el conocimiento en si que contiene un documento patente.

Un reciente trabajo de J. 0. Lanjow y M. Schankerman (2004) plantea este problema y propone la creación de un "indice de calidad de patentes» donde se mediria el número de patentes necesario para crear patentes ajustadas a la calidad, de lo que resultaría una medida más útil de los 
resultados de I+D. Esta nueva aproximación al estudio de las patentes resulta interesante pues parece razonable valorar la calidad antes de la cantidad; los números pueden ser indicativos, pero quizá lo que resulte interesante sea conocer qué es lo que da lugar a estos números. En este sentido Lanjow y Schankerman apuestan en su trabajo por la utilización de cuatro indicadores para medir la calidad: las citaciones posteriores (número de patentes posteriores que citan a una patente determinada durante un periodo de cinco años), las citaciones anteriores (número de patentes citadas previamente en una patente dada), número de solicitudes presentadas de una misma patente, lo que se conoce como la familia de la patente (número de solicitudes presentadas de forma paralela en distintas jurisdicciones). El cuarto de los indicadores, quizá lo más interesante de este trabajo, parte de la constatación de que el peso de estos tres indicadores varía en función del campo tecnológico en el que se adscribe la patente. Por ejemplo en el campo de la industria farmacéutica el indicador clave que indica la calidad de las patentes es el que hace referencia a las citaciones posteriores.

Otro dato relevante es que el estudio de las patentes comienza a salir del campo de la economía para ser objeto de estudio de otras disciplinas. Ejemplo de ello son los interesantes trabajos que se están llevando a cabo desde perspectivas bibliométricas -las que miden el flujo de conocimiento desde la investigación a la aplicación tecnológica-, utilizando como indicadores el número de citas de publicaciones científicas que aparecen en los documentos de patente y que sirven para ilustrar el estado de la técnica en la que se apoya la patente (Albert y Plaza, 2004). Otro campo desde el que se están haciendo trabajos sobre todo de bases de datos sobre patentes de los siglos XVIII, XIX y primeras décadas del XX, es el de la historia de la propiedad industrial. Desde 1999 la Universidad Autónoma de Madrid (UAM) y la Oficina Española de Patentes y Marcas están colaborando en la catalogación y estudio del material que conserva el Archivo Histórico de esta oficina (Sáiz González, 1995, 1996 y 1999).

\section{Qué es una patente}

Desde el análisis filosófico de la política cientifica y tecnológica se viene constatando que se suelen dar carencias en la comprensión de términos y conceptos. Estos defectos de conceptualización justifican una revisión de situaciones que se pueden considerar como bien establecidas. Por ello un repaso sobre cómo ha evolucionado la legislación en el ámbito de las patentes y la influencia que la propia legislación ha ejercido sobre ellas, va a ser el primer tema a tratar.

Con estos trabajos como punto de partida se va a estudiar la patente como fuente de información y formación tecnológica. Después, una aproximación histórica permitirá conocer cómo se han generado las diferentes estructuras administrativas que hacen posible que esta información tecnológica viaje de forma ordenada y se ponga en el mercado. Un paso más en este estudio llevará, trasladando el concepto de calidad frente al de cantidad, a analizar el caso del CSIC y en concreto las solicitudes de patentes del área de biología y biomedicina.

Una patente es un documento que atribuye a su titular un derecho exclusivo de explotación de carácter temporal, 20 años, sobre la invención. La exclusividad está limitada sólo al pais para el que se ha solicitado y concedido la patente. Para que una invención sea patentable, la Ley de Patentes (LP) exige como requisitos básicos que aporte novedad, que tenga actividad inventiva, que pueda ser objeto de aplicación industrial, y que cuente con una descripción suficiente y clara para que un experto en la materia pueda aplicarla. Los tres primeros requisitos, aparentemente claros, presentan también matices que conviene detallar.

Novedad: la LP considera que una invención es nueva cuando lo que aporta no está comprendido en lo que se denomina «el estado de la técnica». Este estado de la técnica lo conforma todo el material que, con anterioridad a la fecha de solicitud de la patente, ha sido hecho público por cualquier medio o soporte. Por ejemplo, pertenecen al estado de la técnica un producto comercializado, una descripción oral seguida de una publicación (la fecha que se tomaria sería la de la exposición oral); por el contrario, una descripción errónea que no permita la consecución de un objetivo, no forma parte de este estado de la técnica. Otro caso que se puede dar es que la invención esté comprendida en otra invención anterior, y que por tanto pertenezca ya al estado de la técnica, pero tenga unas caracteristicas propias que no aparezcan descritas en ese estado de la técnica; si ésta innovación cumple los otros dos requisitos de actividad inventiva y aplicación industrial, será patentable como patente dependiente.

Actividad inventiva: se considera que una invención tiene actividad inventiva si, teniendo en cuenta el estado

ARBOR CLXXXI 715 SEPTIEMBRE-OCTUBRE (2005) 333-350 ISSN: 0210-1963 
de la técnica, no resulta obvia para un experto en la materia.

Aplicación industrial: se considera que una invención es susceptible de aplicación industrial cuando su objeto puede ser fabricado o utilizado en cualquier clase de industria. Por poner algún ejemplo quedan fuera de posible aplicación industrial los métodos de tratamiento quirúrgico o terapéutico y de diagnóstico aplicados al cuerpo humano o animal, aunque no ocurre lo mismo con los productos, sustancias o composiciones, los aparatos o instrumentos necesarios para la puesta en práctica de los métodos de tratamiento o de diagnóstico mencionados.

\section{Legislación y patentes}

La necesidad de contar con una buena legislación de patentes es algo que hoy es entendido y asumido por todos. Su influencia directa en la investigación protegiendo los resultados, así como en la economía, impulsando la innovación tecnológica y elevando el nivel competitivo de la industria, son en principio argumentos de peso. Pero estas circunstancias hoy tan asumidas, hace dieciocho años generaban contradicciones con la legislación entonces vigente. Hasta 1986 España contaba con una legislación de patentes que databa de 1929, el llamado Estatuto de Propiedad Industrial (EPI), obsoleto e imposibilitado para regular aunque fuera un desarrollo tecnológico como el español de entonces. La entrada en la Comunidad Europea obligó, como en otros muchos campos, a hacer compatible la legislación y a incluir normativas ya asumidas por los otros estados miembros. En el caso de las patentes habia que incorporar lo aprobado en el Convenio de Munich de 1973 sobre patente europea y el Convenio de Luxemburgo de15 de diciembre de 1975 relativo a la patente comunitaria. Por lo tanto la Ley de Patentes de 1986 (LP) regula la patentabilidad de las invenciones siguiendo el Derecho europeo y, por primera vez se introduce en España la posibilidad de patentar productos químicos, farmacéuticos y alimentarios, aunque, para el caso de los productos químicos y farmacéuticos, la propia ley estableciera otra demora, hasta el 7 de octubre de 1992, por los problemas que una implantación rápida pudiera ocasionar en los correspondientes sectores industriales.

Un tema importante que aborda la LP es el referido a las invenciones laborales "las invenciones realizadas por el trabajador durante la vigencia del contrato de trabajo o de ser- vicios con la empresa que sean fruto de la actividad de investigación explícita o implícitamente constitutiva del objeto de su contrato, pertenecen al empresarion (título IV, artículo 15). Este artículo sirve de referencia para el resto pues el artículo 20, dedicado específicamente a los investigadores pertenecientes a las administraciones públicas y a los profesores de la universidad, en su apartado 1 remite al artículo 15 mencionado, y en su apartado 2 la Ley atribuye a la universidad la titularidad de las invenciones de los profesores y en los apartados 8 y 9 se deja abierta la posibilidad de aplicar esto mismo a los investigadores de los entes públicos de investigación.

Hay otros dos datos interesantes que introduce la nueva ley; uno es la incorporación en el procedimiento de la concesión de un "informe sobre el estado de la técnican, informe que fortalece las patentes concedidas y que permite, a la hora de elaborar un estudio como el que se pretende, valorar la calidad frente a la cantidad; y el otro es la creación de un fondo documental de patentes de todo el mundo, aunque se hace hincapié en las patentes en lengua española. Hoy dia la Oficina Española de Patentes y Marcas (OEPM) tiene disponibles, para cualquier tipo de consultas, bases de datos nacionales y extranjeras (para invenciones en español OEPMPAT: con datos bibliográficos de documentos de patentes y modelos de utilidad tramitados por el EPI y por la $L P$, asi como las patentes europeas y las solicitadas vía PCT que designan a España y que generan un documento en español; LATIPAT: contiene Patentes y Modelos de dieciocho paises iberoamericanos; CLIPAT: con la Clasificación Internacional de Patentes (CIP); y para invenciones en otros idiomas esp@cenet: contiene los datos bibliográficos de las patentes publicadas en los dos últimos años en cualquier estado miembro de la Organización Europea de Patentes, así como las de la Oficina Europea de Patentes (OEP) y los de la Organización Mundial de la Propiedad Intelectual (OMPI)). Este es un dato relevante pues hoy dia es difícil hablar de innovación sin información tecnológica: el nivel de desarrollo tecnológico será mayor si las estructuras productivas tienen facilidades de acceso a esa información.

\section{La patente como generadora de estructuras para proteger resultados}

Ya se ha hecho referencia al papel tan importante que desempeñan las patentes en el sistema de innovación de cualquier pais. Si partimos de la definición que hace Mikel Bue- 
sa de sistema de innovación como "conjunto de las organizaciones de naturaleza institucional y empresarial que, dentro del territorio correspondiente, interactúan entre si con objeto de asignar recursos a la realización de actividades orientadas a la generación y difusión de los conocimientos sobre los que se soportan las investigaciones ...) (Buesa, 2003: 236), las patentes sirven de nexo de unión, entre las organizaciones implicadas directamente en la obtención del conocimiento (los centros públicos de investigación o las universidades) y las empresas innovadoras que asumen el desarrollo de la investigación, su aplicación productiva y su puesta en el mercado. Pero se daban dos circunstancias que hacian que esta conexión no fuera fluida; la primera era la falta, en el ámbito de la producción científica, de una cultura de protección de resultados científicos antes de su publicación en revistas científicas, y la segunda la no consideración del conocimiento como un bien económico y por lo tanto la carencia de las estructuras necesarias para gestionarlo como tal.

El reconocimiento de estas circunstancias unido al importante papel que adquiere la patente en el nuevo marco legal generado a partir de 1986, pone sobre la mesa la necesidad de crear nuevas estructuras que fomenten la protección de resultados en el ámbito de la investigación pública, o lo que es lo mismo, crear organizaciones que faciliten la relación entre los dos extremos: era necesario establecer los mecanismos que permitieran el flujo de conocimiento e información, la cooperación y la canalización de los recursos. Así desde el primer Plan Nacional de I+D (1988-1991) se impulsó la creación, en universidades y organismos públicos de investigación, de Oficinas de Transferencia de Resultados de Investigación (OTRI) con la idea de facilitar la comunicación y cooperación con las empresas y facilitar así la transferencia de resultados de la investigación pública al sector industrial. Todas estas oficinas quedaban bajo la coordinación de la Oficina de Transferencia de Tecnologia (OTT), unidad dependiente de la Secretaria General del Plan. Sobre los resultados de estas iniciativas estatales en la década de los ochenta ver Represa, Castro, Fernández de Lucio, 2003 y Buesa, 2003.

Pero en este campo no todo era nuevo. Había experiencias anteriores que resultaron de gran utilidad. Por ejemplo en el CSIC funcionaba la Oficina de Valoración y Transferencia de Tecnologia que coordinaba y tramitaba el conocimiento generado en los distintos institutos de este organismo. El CSIC, como heredero de la red de organismos científicos cre- ados por la Junta para Ampliación de Estudios (JAE) y por la Fundación Nacional para Investigaciones Científicas y Ensayos de Reformas (FINICER), aprovechó algunas de las ideas puestas en marcha antes de la guerra -sobre todo de la FINICER pues la JAE hizo más hincapié en la investigación básica- que favorecian la concentración de esfuerzos para mejorar la investigación aplicada. Asi tras la guerra la investigación técnica quedó organizada bajo el Patronato Juan de la Cierva. El objetivo era unificar esfuerzos con el fin de responder a los intereses nacionales. Este mismo objetivo fue el que llevó a que una misma persona, Juan Antonio Suanzes, fuera al tiempo Presidente del Patronato Juan de la Cierva y Presidente del Instituto Nacional de Industria (INI). La coordinación entre una organización y otra no fue una tarea fácil y quizá, estos inicios, marquen ya la tendencia que va a caracterizar la relación entre la investigación y la industria en España. Un estudio muy completo sobre el Patronato Juan de la Cierva es el realizado por Santiago López Garcia $(1997,1998,1999)$.

\section{La patente como fuente de información tecnológica}

Hoy dia son múltiples las vías por las que viaja la información y el conocimiento científico y tecnológico. Congresos científicos, publicaciones, tesis doctorales o exposiciones son medios por todos conocidos por los que cualquier interesado en cualquier materia puede acceder a este conocimiento. Por el contrario, raras veces las patentes son utilizadas de esta forma, algo que no deja de resultar curioso sobre todo si se tiene en cuenta que son unos documentos, que por ley, tienen que hacerse públicos. Una primera aproximación a lo que se conoce como literatura de patente -a priori críptica o excesivamente técnica- permite hacer otro tipo de lectura que da a la patente un protagonismo que hasta ahora no habia tenido.

\section{La forma}

Una de las condiciones fundamentales que debe cumplir una patente es que debe contar con una explicación clara de lo que se quiere patentar. Por ello normalmente esta documentación tiene una pretendida voluntad divulgativa, caracteristica ausente en otras informaciones de carácter más tecnológico; en el resumen que debe incluir todo documento patente normalmente se pueden encontrar datos del con-

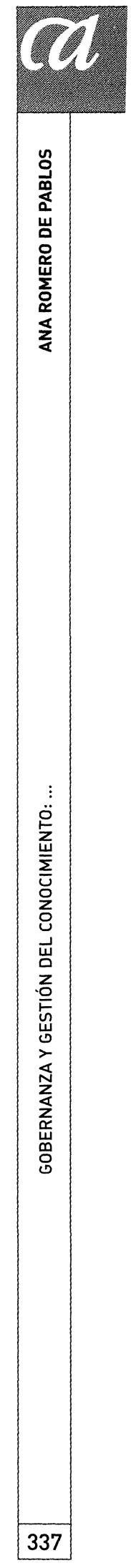

ARBOR CLXXXI 715 SEPTIEMBRE-OCTUBRE (2005) 333-350 ISSN: 0210-1963 
texto en el que se ha creado la investigación, pero también diferentes ejemplos de posibles aplicaciones industriales. Esto es una gran ventaja, pero no la única. Por ejemplo el documento patente suele tener una estructura bastante uniforme, lo que también facilita el estudio; además cubre, en principio, todos los sectores técnicos que, siendo susceptibles de aplicación industrial, puedan resultar relevantes tanto en el ámbito nacional como en el internacional. Otra particularidad destacable es que estos documentos contienen una información que raramente se difunde en otros formatos que no sea la patente, por lo que resultan no solo complementarios sino necesarios para hacer un estudio en profundidad. Un último dato que también facilita el acercamiento a lo que se conoce como «literatura de patentes", es que desde 1971 todas las oficinas nacionales responden a una única ordenación, la Clasificación Internacional de Patentes (CIP).

En un mismo documento patente están incluidos diferentes tipos de documentos, todos ellos públicos, que varian en función de la legislación de cada pais. Hay tres básicos e imprescindibles: la solicitud, la concesión y el informe sobre el estado de la técnica. La solicitud de la patente, único documento hecho por el propio inventor y que él mismo presenta ante una Oficina de Patentes, tiene un doble interés. Por un lado es donde se recoge la primera descripción de la invención, pero además los propios términos y delimitaciones que en él se establecen, son muy indicativos de lo que busca proteger el inventor. El documento de concesión de la patente también describe la invención, pero a diferencia de la solicitud, lo hace tal y como va a ser protegida a partir de entonces. Hasta llegar a la concesión de una patente son diferentes las fases, en función del procedimiento elegido por el solicitante, por las que una solicitud debe pasar; normalmente, durante estas fases, la solicitud original sufre modificaciones que se le van añadiendo. El informe sobre el estado de la técnica, que hasta 1986 no se contemplaba en España, es un documento redactado por las oficinas de patentes que reúne la relación de todo lo publicado, ya sean patentes o no, que tenga algún tipo de vínculo con la solicitud en cuestión. Este informe da por tanto a conocer los elementos del estado de la técnica necesarios a tener en cuenta con el objeto de determinar la novedad y actividad inventiva de la solicitud de la patente, y permite, tanto al solicitante como a cualquier otra persona interesa$\mathrm{da}$, valorar y apreciar en su justa medida su valor. Al ser un documento con tanto peso específico, pues no sólo de él depende la concesión o no de la patente sino, como se indi- ca a continuación, resulta clave a la hora de generar y transmitir conocimiento, las oficinas de patentes tienen perfectamente establecido cómo proceder para realizarlos: se estudia la solicitud, se determina el objeto de la invención, se plantea la estrategia de búsqueda en las distintas bases de datos, se solicitan y estudian los documentos seleccionados en la búsqueda anterior y por último se seleccionan y valoran dichos documentos.

Si desde el punto de vista jurídico el documento importante es el de la concesión, desde el punto de vista científico, tecnológico y de la política científica, tienen mayor importancia la solicitud, primer documento en dar a conocer el contenido de la invención, y el informe del estado de la técnica por lo que aporta sobre la novedad y sobre el estado de la técnica en el que se encuadra.

\section{El contenido}

Son muchos los usos de la información contenida en las patentes y esta información sin duda no es de exclusiva utilización por los científicos o por los técnicos. Obviando el uso de patentes con fines legales, en principio se pueden establecer dos ámbitos claros de aplicación: el primero donde hay un uso inmediato de la tecnología patentada como fuente de ayuda imprescindible a la $I+D$, y el segundo, donde un uso más reposado, analítico y a más largo plazo, permite que la industria y las administraciones públicas planifiquen actividades futuras y opten por estrategias a seguir. Así la información contenida en las patentes permite estudiar:

- El estado de la técnica de un sector tecnológico determinado. Un buen conocimiento de lo que ocurre no solo permite no duplicar esfuerzos, sino que también sirve de acicate; permite solucionar problemas tecnológicos específicos, conocer en qué estado están los posibles competidores y valorar la oportunidad de hacer inversiones.

- El seguimiento de la evolución tecnológica que se ha producido, cuáles han sido las tendencias, cómo han sido los tiempos.

- La transferencia tecnológica: cómo viaja la tecnología, cómo se establecen las relaciones entre las partes interesadas, si hay intermediarios, cómo se produce la aportación del capital, si esta transferencia de tecnologia implica formación de personal, si se producen modificaciones en la forma de hacer ciencia, en los 
métodos de gestión, las instituciones u organismos implicados ...

En definitiva el documento patente parece un instrumento interesante de estudio a la hora de evaluar la creación y divulgación del conocimiento científico y tecnológico de un pais, y en el caso concreto del CSIC, permiten ver el papel desempeñado por este organismo en las redes de intercambio de conocimiento no sólo en España, sino también a nivel internacional, así como los efectos y consecuencias que han tenido en el CSIC la producción de patentes.

\section{Procedimientos de solicitud}

Los distintos procedimientos que contempla y regula la legislación sobre patentes aportan también mucha información a la hora de estudiarlas. Las opciones por seguir un procedimiento y no otro, revelan no sólo la estrategia adoptada para su aprobación, sino también las expectativas depositadas en esas solicitudes. Se puede hacer una valoración de la patente en función del procedimiento que ha seguido: una solicitud que inicia un procedimiento nacional, y luego continúa con otros procedimientos internacionales, lleva a pensar, en principio, en una patente prometedora, fuerte. Las diferencias establecidas en las distintas legislaciones que rigen los procedimientos pueden también marcar tendencias o costumbres, diferenciadas por territorios, a la hora incluso de optar o no por patentar. Todos estos matices llevan a pensar que un estudio que contemple sólo datos cuantitativos o estadisticos de patentes, tendria un resultado cuando menos incompleto.

De forma general se pueden establecer tres tipos de sistemas de concesión de patentes en el mundo. De menor o mayor rigor, el primero es el llamado sistema de simple depósito. En el que tan sólo se examinan las formalidades administrativas, carece por tanto de la información tecnológica necesaria y no aporta ninguna certidumbre sobre la validez de la patente. El segundo es el sistema con informe sobre el estado de la técnica, tiene como ventaja que hace pública, como ya se ha mencionado, una información tecnológica de calidad, que clarifica el mercado aportando asi mayor certidumbre de validez que el sistema anterior. El sistema con examen previo es el que garantiza un mayor rigor de estudio pues incluye un informe sobre la novedad y la actividad inventiva de la solicitud.
Para dar una idea de la complejidad que hay detrás de los procedimientos de solicitud, se exponen a continuación los pasos que con mayor frecuencia tiene que dar la Oficina de Transferencia de Tecnología del CSIC, oficina dedicada a la tramitación de las solicitudes de patentes generadas por los investigadores de este organismo, para conseguir la aprobación de una patente. La solicitud nacional, la solicitud internacional y la solicitud europea, son tres procedimientos con ámbitos distintos de actuación aunque semejantes en algunas de sus fases y sobre todo en los objetivos; los tres son independientes y lo interesante es que pueden ser complementarios.

\section{Solicitud nacional}

Los requisitos mínimos para que una solicitud sea admitida a trámite y obtenga así una fecha de presentación son: presentar una declaración que aclare por qué se solicita la patente, contar con una identificación clara del solicitante, dar un título a la invención, aportar una descripción así como una o varias reivindicaciones, abonar la tasa de solicitud y establecer la prioridad (el pais de origen y la fecha).

Del examen formal y técnico interesa destacar que si la invención que se quiere patentar se refiere a materia biológica, está obligado el solicitante siguiendo el Tratado de Budapest, a hacer un depósito de una muestra de dicha materia en la institución nacional reconocida para ello. En el caso español ésta institución es la Universidad de Valencia, que acoge la llamada "Colección española de cultivos tipon (CECT) ${ }^{1}$. Otro papel fundamental que realiza este examen es el de delimitar lo que se entiende por invención, por ejemplo los descubrimientos o las teorias científicas o los programas de ordenador no son considerados invenciones; la ley establece lo que no es susceptible de aplicación industrial: métodos de tratamiento quirúrgico o terapéuticos, métodos de diagnóstico aplicados al cuerpo humano o animal, aunque por el contrario sí lo son los productos, aparatos o instrumentos necesarios para la aplicación de estos métodos; y por último deja fuera de posible patente todas las invenciones cuya explotación pueda ir contra el orden público o la moral y las buenas costumbres. En este grupo, hoy un tema de plena actualidad, se incluyen los procedimientos de clonación de seres humanos, los de modificación genética germinal del ser humano, la utilización de embriones con fines industriales o comerciales, o los procedimientos que modifican la identidad genética de animales sin una

ARBOR CLXXXI 715 SEPTIEMBRE-OCTUBRE (2005) 333-350 ISSN: 0210-1963 
clara utilidad médica; tampoco son patentables las variedades vegetales, las razas animales, o los procedimientos biológicos de obtención de animales o vegetales.

Sobre el informe del estado de la técnica hay que destacar que lo realizan, en el seno de las propias oficinas de patentes, los técnicos destinados para ello y que una vez finalizado la ley obliga a hacerlo público en los boletines de las oficinas; la OEPM cuenta con su propio boletín: el Boletín Oficial de Patentes Industriales (BOPI).

\section{Solicitud internacional: Tratado de Cooperación en materia de Patentes (PCT)}

Aunque es un tratado que se firmó en Washington en 1970 y en junio de 1978 ya formaban parte de él dieciocho estados, no fue hasta 1989 cuando se produjo la adhesión española entrando en vigor en noviembre de 1989.

Este sistema, normalmente utilizado por el CSIC para poner sus patentes en el mercado internacional, tiene grandes ventajas pues facilita enormemente el procedimiento de solicitud en el ámbito internacional. Pero conviene aclarar que, a diferencia de los otros dos procedimientos tratados, el sistema PCT, no finaliza con la concesión o denegación de una patente, sino que se limita a tramitar y a gestionar las solicitudes.

Las ventajas que aporta el sistema PCT son varias. La primera y más obvia es la internacionalización del proceso además de facilitar algunas fases del procedimiento. Otro punto interesante es la creación de un sistema descentralizado ya que incorpora diferentes oficinas de propiedad industrial de los diferentes paises miembros con el fin de que cada una de ellas, aporte trabajos de forma organizada. Para explicar esto puede servir de ejemplo el caso español. Desde septiembre de 1993, la OEPM cuenta con la condición de administración internacional de búsqueda; en 1999 la OEPM firmó un Acuerdo con la Oficina Europea de Patentes de armonización en materia de búsqueda de PCT. En el 2001 la OEPM obtuvo la Condición de Oficina Internacional de Examen comenzando esta actividad en junio de 2003.

Los pasos que requiere el sistema PCT son los siguientes:

- Solicitud de patente nacional en la oficina local siguiendo la ley nacional.

ARBOR CLXXXI 715 SEPTIEMBRE-OCTUBRE (2005) 333-350 ISSN: 0210-1963
- Pasados 12 meses se presenta, en la misma oficina local, la solicitud PCT que debe incluir los Estados o paises donde interesa extender el trámite.

- Comienza la fase de búsqueda internacional, de publicación y de examen preliminar.

- Si los solicitantes siguen interesados en obtener las patentes deben, en la oficina nacional afectada, pagar las tasas correspondientes $y$, en ocasiones, presentar una traducción.

- Después cada oficina nacional, siguiendo su procedimiento nacional de concesión, procederá a conceder o a desestimar la solicitud.

Asi el sistema PCT establece dos fases claramente diferenciadas, la internacional que comprende la presentación de solicitud internacional, la búsqueda internacional, la publicación internacional y el examen preliminar internacional si así lo quiere el solicitante, y una segunda fase la nacional / regional ante las oficinas elegidas. Desde la primera solicitud nacional ante la oficina local hasta la entrada en las fases nacionales de los distintos paises interesados pueden llegar a transcurrir, en el mejor de los casos, hasta 30 meses. Esta dilatación en el tiempo puede favorecer, pues es aprovechado por los solicitantes para tantear el mercado y buscar las mejores opciones para la patente. Pero en otras ocasiones puede ser un inconveniente: se dan casos de empresas que mantienen el coste económico que supone el trámite de la solicitud, por el interés que tienen en su posible explotación.

\section{Solicitud europea: Convenio de la Patente Europea (CPE-EPC)}

Las solicitudes de patentes para Europa se realizan bajo el Sistema del Convenio de la Patente Europea (CPE-EPC). Este convenio internacional concede una patente de forma centralizada para los paises miembros. Se firmó en octubre de 1973 aunque no entró en vigor, para el primer grupo de países firmantes, hasta octubre de 1977. La adhesión de España se produjo en julio de1986, haciéndose efectivo en octubre del mismo año. Hoy dia forman parte del CPE 29 estados.

Este procedimiento de solicitud es bastante similar al español aunque con una diferencia importante. La recepción de las solicitudes puede hacerse en las oficinas receptoras de cada pais miembro, pero la tramitación se lleva a cabo en la Oficina Europea de Patentes (OEP-EPO) con sedes en Munich y La Haya. 
El sistema CPE comprende dos trámites bien diferenciados, uno previo a la concesión y otro posterior. Del primero forman parte la presentación de la solicitud con la designación de los países donde se quiere proteger la invención, la elaboración del informe del estado de la técnica, la publicación de la solicitud y del informe del estado de la técnica (generalmente en un plazo no superior a los 18 meses de la fecha de solicitud o de prioridad), el examen y la eventual concesión de la patente. EI segundo de los trámites, que se pone en marcha una vez concedida la patente, incluye la posibilidad de que terceros, durante un plazo de 9 meses desde la fecha del anuncio de la concesión, se opongan a ésta (aqui la diferencia con el procedimiento español), y la validación de la patente europea en cada pais seleccionado, normalmente tres meses después de la fecha en que se hace pública la concesión.

La diferencia con el procedimiento español es que en el europeo la oposición de terceros se produce una vez concedida la solicitud de patente, y no previamente al examen como ocurre en el español. Este dato es importante a la hora de estudiar una patente europea; si la oposición de terceros ocasiona modificaciones a la patente concedida, provoca la necesidad de una nueva validación, lo que puede demorar y encarecer el proceso pero al tiempo puede resultar más ágil pues presume la inocencia.

\section{Las Patentes del CSIC}

Ya se ha mencionado que lo que se quiere es utilizar los datos que custodia la Oficina de Transferencia de Tecnología del CSIC para conocer cómo circula la información y el conocimiento científicos contenidos en estos documentos. Para ello en primer lugar, con el fin de presentar un panorama amplio de lo que ocurre en las distintas áreas de investigación del CSIC, se presentan unas tablas ${ }^{2}$ con distintos datos útiles para contextualizar el estudio de las patentes del área de biología y biomedicina.

\section{Solicitudes nacionales}

De las cuatro tablas (Cuadros 1 a 4) que hacen referencia a los datos de las solicitudes, en el ámbito nacional, de las patentes del CSIC, tiene interés destacar en primer lugar el incremento constante que se produce durante la década estudiada y sobre todo el pico del año 2002 (Cuadro 1). Si a estos datos se incorpora una variable tan importante como son las áreas de conocimiento en donde se adscriben estas posibles patentes (Cuadro 2), en cifras totales destacan las áreas de Ciencia y Tecnologías Químicas, la de Biologia y Biomedicina y la de Ciencia y Tecnologias de Materiales. Los datos de la evolución de las solicitudes por años (Cuadro 3) indican que el "despegue" se produce antes en el área de las Ciencia y Tecnologías Quimicas, en 1995, que en la de Biología y Biomedicina, que se da en torno al 2000-2001. Pero si se puede hablar de un cambio de tendencia importante en esta área, Biología y Biomedicina, atendiendo a los datos de la tabla que recoge las solicitudes agrupadas por quinquenios (Cuadro 4). En la segunda mitad de la década se dobla el número de solicitudes, lo que no ocurre en ninguna de las otras áreas.

CuAdro 1. Total de solicitudes nacionales del CSIC (1994-2004)

\begin{tabular}{|c|c|}
\hline Año & Solicitudes nacionales \\
\hline 1994 & 34 \\
\hline 1995 & 36 \\
\hline 1996 & 54 \\
\hline 1997 & 63 \\
\hline 1998 & 69 \\
\hline 1999 & 93 \\
\hline 2000 & 97 \\
\hline 2001 & 99 \\
\hline 2002 & 119 \\
\hline 2003 & 95 \\
\hline 2004 & 83 \\
\hline
\end{tabular}


CUADRo 2. Total de solicitudes nacionales del CSIC por áreas (1994-2004)

\begin{tabular}{|l|c|}
\hline \multicolumn{1}{|c|}{ Áreas } & Total solicitudes nacionales \\
\hline Humanidades y Ciencias Sociales & 0 \\
\hline Biología y Biomedicina & 174 \\
\hline Recursos Naturales & 33 \\
\hline Ciencias Agrarias & 52 \\
\hline Ciencia y Tecnologías Físicas & 114 \\
\hline Ciencia y Tecnología de Materiales & 122 \\
\hline Ciencia y Tecnología de Alimentos & 117 \\
\hline Ciencia y Tecnologias Químicas & 229 \\
\hline
\end{tabular}

CUADRo 3. Solicitudes nacionales del CSIC por áreas y años (1994-2004)

\begin{tabular}{|c|c|c|c|c|c|c|c|c|c|c|c|}
\hline Áreas $^{3}$ & 1994 & 1995 & 1996 & 1997 & 1998 & 1999 & 2000 & 2001 & 2002 & 2003 & $2004^{4}$ \\
\hline 1 & & & & & & & & & & & \\
\hline 2 & 2 & 4 & 6 & 11 & 16 & 18 & 18 & 27 & 27 & 20 & 25 \\
\hline 3 & 2 & 2 & 4 & 2 & 2 & 2 & 6 & 4 & 5 & 7 & 4 \\
\hline 4 & 7 & 1 & 3 & 2 & 3 & 8 & 3 & 6 & 7 & 18 & 5 \\
\hline 5 & 6 & 6 & 10 & 9 & 4 & 5 & 10 & 12 & 22 & 12 & 12 \\
\hline 6 & 4 & 6 & 8 & 13 & 13 & 13 & 16 & 14 & 17 & 11 & 6 \\
\hline 7 & 8 & 1 & 10 & 9 & 10 & 22 & 16 & 10 & 7 & 27 & 13 \\
\hline 8 & 5 & 16 & 13 & 17 & 21 & 25 & 28 & 26 & 34 & 20 & 17 \\
\hline
\end{tabular}

CUADRo 4. Solicitudes nacionales del CSIC por áreas y diferenciadas por quinquenios (1994-2004)

\begin{tabular}{|l|c|c|}
\hline \multicolumn{1}{|c|}{ Áreas } & 1994-1999 & 2000-2004 \\
\hline Humanidades y Ciencias Sociales & & \\
\hline Biología y Biomedicina & 57 & 117 \\
\hline Recursos Naturales & 14 & 19 \\
\hline Ciencias Agrarias & 24 & 28 \\
\hline Ciencia y Tecnologías Físicas & 40 & 74 \\
\hline Ciencia y Tecnología de Materiales & 57 & 65 \\
\hline Ciencia y Tecnología de Alimentos & 60 & 57 \\
\hline Ciencia y Tecnologías Químicas & 97 & 132 \\
\hline
\end{tabular}

\section{Solicitudes PCT}

Con respecto a los datos de las solicitudes PCT parece lógico conocer en primer lugar el total de las solicitudes nacionales que en principio pueden despertar mayor interés en el ámbito internacional ${ }^{5}$ (Cuadro 5). Fue en los años 2001 y 2002 cuando se obtuvo el mejor resultado, por encima del cincuenta por ciento. Pero este dato, aunque interesante, conviene matizarlo más. Para ello en el Cuadro 6 se han agrupado, por áreas, por un lado el total de solicitudes nacionales y por otro el total de las PCT; el objeto es ver si las tendencias que se producen en el ámbito nacional, se dan también en el internacional. Los datos muestran similitudes pero también algunas diferencias. Por ejemplo vemos 
que, a diferencia de lo que ocurre para el caso de las nacionales, solicitudes adscritas a áreas como Tecnologia de Alimentos, Tecnologías Físicas y Recursos Naturales tienen, en este caso, una presencia mayor con unos porcentajes más próximos a los de las solicitudes adscritas a áreas de Tecnologias Quimicas o de Biología y Biomedicina. Esto lleva a plantear otra cuestión: ¿se puede hablar de áreas con mayor costumbre o tradi- ción a la hora de patentar en el extranjero?, ¿hay comunidades científicas con hábitos de internacionalización más acentuados? (sobre estos temas hay ya trabajos interesantes; para el caso de la comunidad bioquímica y biomédica española ver Santesmases y Muñoz, 1997); estas preguntas son un ejemplo de las muchas que pueden surgir a la hora de intentar entender qué hay detrás de los números y porcentajes.

CUADRo 5. Solicitudes nacionales que pasan al sistema PCT

\begin{tabular}{|c|c|c|c|}
\hline Año & Solicitudes nacionales & Solicitudes PCT & $\%$ PCT \\
\hline 1994 & 34 & 3 & $8,8 \%$ \\
\hline 1995 & 36 & 13 & $36,1 \%$ \\
\hline 1996 & 54 & 13 & $24,07 \%$ \\
\hline 1997 & 63 & 16 & $25,3 \%$ \\
\hline 1998 & 69 & 15 & $21,7 \%$ \\
\hline 1999 & 93 & 28 & $30,10 \%$ \\
\hline 2000 & 97 & 46 & $47,4 \%$ \\
\hline 2001 & 99 & 62 & $62,6 \%$ \\
\hline 2002 & 119 & 73 & $61,3 \%$ \\
\hline 2003 & 95 & 38 & $40 \%$ \\
\hline
\end{tabular}

\section{CUADRo 6. Solicitudes PCT repartidas por áreas}

\begin{tabular}{|l|c|c|c|}
\hline \multicolumn{1}{|c|}{ Áreas } & Total solicitudes & Solicitudes PCT & $\%$ PCT \\
\hline Humanidades y Ciencias Sociales & 0 & 0 & \\
\hline Biología y Biomedicina & 174 & 64 & $36,7 \%$ \\
\hline Recursos Naturales & 33 & 12 & $36,6 \%$ \\
\hline Ciencias Agrarias & 52 & 11 & $21,1 \%$ \\
\hline Ciencia y Tecnologías Fisicas & 114 & 42 & $36,8 \%$ \\
\hline Ciencia y Tecnología de Materiales & 122 & 33 & $27,04 \%$ \\
\hline Ciencia y Tecnología de Alimentos & 117 & 44 & $37,6 \%$ \\
\hline Ciencia y Tecnologías Químicas & 229 & 104 & $45,4 \%$ \\
\hline
\end{tabular}

\section{Solicitudes europeas}

El tercer paso en el procedimiento es la tramitación de la solicitud europea. Interesa ver la evolución que han tenido las solicitudes de las patentes del CSIC desde su inicio en el ámbito nacional, su tramitación internacional y su gestión para Europa. Los datos que aqui se exponen (Cuadro 7) sólo permiten un análisis cualitativo, que para comenzar, no deja de ser significativo. Conviene aclarar también, para no hacer una lectura equivocada de los datos, que debido a los tiempos que requieren cada uno de los tres procedimientos de solicitud mencionados, no quiere decir, por ejemplo siguiendo los datos de 1995, que los 3 trámites de solicitudes europeas que inicia el CSIC en ese año, correspondan con algunas de las 36 solicitudes nacionales de ese mismo año. En este caso hay que hacer una lectura más en vertical o por columnas, que horizontal; aunque esta última tiene también importancia pues permite ver una realidad en cuanto al 
número de solicitudes de patentes que el CSIC pone en el mercado europeo.

Con respecto a las áreas de conocimiento (Cuadro 8) parece lógico pensar que la tendencia debe ser muy parecida, sino igual, a lo que muestran los datos relativos a las solicitudes PCT. Pero con esta tabla se quiere también mostrar otro ejemplo o modelo de análisis que permiten estos datos. Ver si, durante el periodo cronológico elegido, se puede hablar de mayor tradición o cultura en materia de patentes, en función de las áreas de conocimiento, a la hora, en este caso, de iniciar la tramitación de solicitudes europeas. Los datos indican que donde la tradición es mayor, al margen de la cantidad, es en el área de Ciencias y Tecnologias Quimicas seguida del área de Biología y Biomedicina. Este dato sería interesante estudiarlo y ver qué es lo que ocurre en estas mismas áreas en la industria española.

CUADRO 7. Evolución de las solicitudes del CSIC en función de los procedimientos seguidos

\begin{tabular}{|c|c|c|c|}
\hline Año & Solicitudes Nacionales & Solicitudes PCT & Solicitudes europeas \\
\hline 1994 & 34 & 3 & 0 \\
\hline 1995 & 36 & 13 & 3 \\
\hline 1996 & 54 & 13 & 4 \\
\hline 1997 & 63 & 16 & 4 \\
\hline 1998 & 69 & 15 & 7 \\
\hline 1999 & 93 & 28 & 7 \\
\hline 2000 & 97 & 46 & 18 \\
\hline 2001 & 99 & 62 & 15 \\
\hline 2002 & 119 & 73 & 18 \\
\hline 2003 & 95 & 38 & 3 \\
\hline 2004 & 83 & 3 & 0 \\
\hline
\end{tabular}

CUADRO 8. Distribución de las solicitudes europeas por áreas

\begin{tabular}{|l|c|l|l|l|l|l|l|l|l|l|}
\hline Año & Solicitudes europeas & $\mathbf{1}$ & $\mathbf{2}$ & $\mathbf{3}$ & $\mathbf{4}$ & $\mathbf{5}$ & $\mathbf{6}$ & $\mathbf{7}$ & $\mathbf{8}$ & Áreas \\
\hline 1994 & 0 & & & & & & & & \\
\hline 1995 & 3 & & & & & & 1 & 2 & \\
\hline 1996 & 4 & & 1 & & & & & & 3 \\
\hline 1997 & 4 & & & & & 1 & & 7 & 2 \\
\hline 1998 & 7 & & 1 & & & 1 & 2 & & 3 \\
\hline 1999 & 7 & & & & 1 & 2 & & & 4 \\
\hline 2000 & 18 & & 5 & & & 2 & & & 11 \\
\hline 2001 & 15 & & 3 & 1 & & & 1 & 3 & 7 \\
\hline 2002 & 18 & & 5 & & & & & 2 & 11 \\
\hline 2003 & 3 & & 2 & & & & & & 1 \\
\hline 2004 & 0 & & & & & & & & \\
\hline
\end{tabular}

\section{Las patentes del área de Biología y Biomedicina del CSIC}

La última parte del capitulo se va a dedicar, ya centrados en el área de Biología y Biomedicina, a plantear el modelo que se defiende como objeto de trabajo. Para conocer qué es lo que ocurre con el conocimiento que se solicita patentar, cómo se gesta, cómo viaja, quién lo gestiona y administra, quién se beneficia, se entiende que no sólo en términos económicos, se han elegido tres cuestiones que permiten ade- 
más evaluar el conocimiento contenido en el documento patente.

La primera de las cuestiones es la que se refiere a la titularidad y autoría de la patente. Interesa ver si el conocimiento se ha gestado en el seno de un único grupo de investigación o si por el contrario ha sido generado de forma conjunta y compartida por grupos de investigación con adscripciones institucionales diferentes (universidad, empresas, centros de investigación extranjeros, etc.). En principio, un trabajo realizado de forma conjunta por grupos de investigación adscritos a diferentes centros o instituciones, parece razonable pensar que cuenta con un valor intrinseco que lo hace más interesante que por ejemplo una patente surgida de un solo grupo de investigación.

La segunda cuestión es la relacionada con la internacionalización del conocimiento. Una forma de valorar el conocimiento es conocer el grado de aceptación que puede llegar a tener en el mercado internacional. Los distintos procedimientos de solicitud de patentes que contempla la legislación y que ya han sido explicados, aportan mucha información. Las opciones elegidas en cada caso por seguir un procedimiento y no otro indican no sólo la estrategia diseñada, en este caso por el CSIC, para su puesta en el mercado, sino también, y esto es importante, las expectativas depositadas en esas solicitudes. Los datos dan a conocer no sólo si interesa o no este conocimiento, sino también las distintas direcciones y caminos que recorre.

Por último, la tercera de las cuestiones que ilustra el modelo de estudio que se quiere plantear es la utilización que se hace de ese conocimiento. Cuántas de estas patentes hay en explotación y en qué condiciones se está produciendo.

Los datos que se incluyen en las tablas que se exponen a continuación, son datos preliminares que habrá que matizar en investigaciones posteriores. Pero se ha considerado pertinente su incorporación pues ponen sobre la mesa la complejidad misma del estudio.

Las dos primeras tablas (Cuadros 9 y 10) recogen los datos de las solicitudes de patentes hechas, entre 1994 y 2004, por los distintos centros del CSIC adscritos al área de Biología y Biomedicina. En la primera de ellas se aprecia el reparto de solicitudes por centros y en la segunda se han agrupado por comunidades autónomas de forma que se puede ver un peso de Madrid y de Cataluña sobre el resto de comunidades ${ }^{6}$. La razón de este "doble centralismo" es obvia, pero quizá sí seria interesante no sólo comparar los datos de la distribución por comunidades y solicitudes de patentes con el número de centros de cada comunidad, sino también ver qué tipo de consecuencias, además de las evidentes, ha tenido y está teniendo esta situación.

CuAdro 9. Solicitudes de patentes de los centros del área de Biología y Biomedicina del CSIC

\begin{tabular}{|l|r|}
\hline \multicolumn{1}{|c|}{ Centro } & Patentes \\
\hline C. Biología Molecular "Severo Ochoa" (CBM) & 159 \\
\hline C. Investigación y Desarrollo (CID) & 101 \\
\hline C. Investigaciones Biológicas (CIB) & 168 \\
\hline C. Nacional de Biotecnología (CNB) & 260 \\
\hline I. de Biología Molecular y Celular de plantas "Primo Yúfera" (IBMCP) & 19 \\
\hline I. de Biología Molecular de Barcelona (IBMB) & 24 \\
\hline I. de Biomedicina de Valencia (IBV) & 15 \\
\hline I. de Bioquímica Vegetal y Fotosíntesis (IBVF) & 21 \\
\hline I. de Investigaciones Biomédicas "Alberto Sols" (IIB) & 62 \\
\hline I. de Investigaciones Biomédicas de Barcelona (IIBB) & 2 \\
\hline I. de Microbiología Bioquímica (IMB) & 13 \\
\hline I. de Neurobiología "Ramón y Cajal" (INRC) & 61 \\
\hline I. de Parasitología y Biomedicina "López Neyra" (IPBLN) & 7 \\
\hline
\end{tabular}




\begin{tabular}{|l|r|}
\hline \multicolumn{1}{|c|}{ Comunidad Autónoma } & \multicolumn{1}{c|}{ Patentes } \\
\hline Andalucía & 28 \\
\hline Cataluña & 127 \\
\hline Madrid & 710 \\
\hline Castilla y León & 13 \\
\hline C. Valenciana & 34 \\
\hline
\end{tabular}

\section{La autoría}

La autoria o titularidad de las solicitudes de patentes es la primera de las cuestiones que se quiere abordar y que ejemplifica este nuevo modelo de estudio. Los datos que se muestran a continuación (Cuadros 11 a 14) son los relativos a las solicitudes de patentes realizadas por los cuatro primeros centros que encabezan el Cuadro 9. Esto tiene el inconveniente de que son sólo centros de Madrid y Barcelona, pero la ventaja es que proporcionan un número de solicitudes razonable para explicar el estudio que se pretende abordar.

Con el objeto de ver cómo y quiénes han gestado el conocimiento científico y técnico que ha dado lugar a estas solicitudes de patentes, se han establecido diferencias en función de la distinta autoría que figura en el documento patente. Quizá aqui interese aclarar que al hablar de autorías se está hablando de los centros a los que están adscritos los grupos de investigación o los inventores. Las entradas que se han establecido son:

- solicitudes propias: las que tienen sólo como inventores a grupos de investigación adscritos al mismo instituto del CSIC que solicita la patente

- $\quad$ solicitudes con otro centros del CSIC: las que entre los solicitantes figuran grupos de investigación de diferentes centros del CSIC

- solicitudes con otros centros de investigación: las solicitudes que tienen como inventores a grupos de investigación adscritos a un centro concreto del CSIC y a otros grupos pertenecientes a otros centros de investigación españoles

- solicitudes con centros de investigación extranjeros: solicitudes que tienen como inventores a grupos de investigación adscritos a un centro concreto del CSIC y a otros grupos pertenecientes a otros centros de investigación pero en este caso extranjeros
- solicitudes con universidades: las solicitudes que tienen como inventores a grupos de investigación adscritos a un centro concreto del CSIC y a grupos de investigación adscritos a cualquier universidad española

- solicitudes con universidades extranjeras: las solicitudes que tienen como inventores a grupos de investigación adscritos a un centro concreto del CSIC y a grupos de investigación adscritos a cualquier universidad extranjera

- solicitudes con empresas: las solicitudes que tienen como inventores a grupos de investigación adscritos a un centro concreto del CSIC y a otros grupos pertenecientes empresas españolas

- solicitudes con empresas extranjeras: las que tienen como inventores a grupos de investigación adscritos a un centro concreto del CSIC y a otros grupos pertenecientes a empresas en este caso extranjeras

Un estudio detallado de estos datos va a permitir conocer cuáles son los centros del CSIC en esta área que producen mayor conocimiento científico y tecnológico, pero también cómo es este conocimiento. Cómo se ha generado, qué caracteristicas tiene el grupo de investigación, cuáles son sus componentes, qué relaciones se han creado con otros centros y grupos investigadores, si estas relaciones eran previas o si las ha ocasionado el propio trabajo de investigación. Otra información importante que estos datos pueden proporcionar es la procedencia de la financiación económica necesaria para generar ese conocimiento: pública, privada, nacional o extranjera. En los casos de solicitudes de patentes conjuntas seria interesante ver también de donde ha partido la iniciativa a la hora de la puesta en marcha de la investigación. Esto último cobra una importancia relevante en los casos en los que la solicitud de la patente ha sido hecha de forma conjunta por investigadores del CSIC y el mundo de la empresa. 
CUADRo 11. Titularidad de las solicitudes del C. Biologia Molecular "Severo Ochoa"

\begin{tabular}{|l|l|c|}
\hline CBM & Autoría & Patentes \\
\hline & Propias & 89 \\
\hline & Con centros del CSIC & 45 \\
\hline & Con otros centros de investigación & 5 \\
\hline & Con centros de investigación extranjeros & \\
\hline & Con la universidad & 12 \\
\hline & Con universidades extranjeras & \\
\hline & Con empresas & 3 \\
\hline & Con empresas extranjeras & 5 \\
\hline
\end{tabular}

CUADRO 12. Titularidad de las solicitudes del C. Investigación y Desarrollo

\begin{tabular}{|l|l|c|}
\hline CID & Autoría & Patentes \\
\hline & Propias & 55 \\
\hline & Con centros del CSIC & 10 \\
\hline & Con otros centros de investigación & \\
\hline & Con centros de investigación extranjeros & 2 \\
\hline & Con la universidad & 2 \\
\hline & Con universidades extranjeras & \\
\hline & Con empresas & 28 \\
\hline & Con empresas extranjeras & 1 \\
\hline
\end{tabular}

CuAdro 13. Titularidad de las solicitudes del C. Investigaciones Biológicas

\begin{tabular}{|l|l|c|}
\hline CIB & Autoría & Patentes \\
\hline & Propias & 84 \\
\hline & Con centros del CSIC & 25 \\
\hline & Con otros centros de investigación & 3 \\
\hline & Con centros de investigación extranjeros & 17 \\
\hline & Con la universidad & 25 \\
\hline & Con universidades extranjeras & 5 \\
\hline & Con empresas & 7 \\
\hline & Con empresas extranjeras & \\
\hline
\end{tabular}

CuAdro 14. Titularidad de las solicitudes del C. Nacional de Biotecnología

\begin{tabular}{|l|l|c|}
\hline CNB & Autoria & Patentes \\
\hline & Propias & 148 \\
\hline & Con centros del CSIC & 38 \\
\hline & Con otros centros de investigación & 1 \\
\hline & Con centros de investigación extranjeros & 23 \\
\hline & Con la universidad & 7 \\
\hline & Con universidades extranjeras & \\
\hline & Con empresas & 35 \\
\hline & Con empresas extranjeras & 7 \\
\hline
\end{tabular}

ARBOR CLXXXI 715 SEPTIEMBRE-OCTUBRE (2005) 333-350 ISSN: 0210-1963 


\section{La internacionalización}

El segundo de los criterios que se propone para conocer el grado de aceptación que pueda o no tener el conocimiento contenido en un documento patente, es estudiar el procedimiento que ha seguido para su puesta en el mercado. Los distintos procedimientos y las complejidades que tienen cada uno de ellos ya han quedado ampliamente explicados más arriba. Pero ahora es interesante ver cómo puede repercutir el seguir un procedimiento u otro. El procedimiento indica finalmente un recorrido geográfico del conocimiento cientifico y tecnológico contenido en el documento patente. Al utilizar datos que hablan de solicitudes de patentes y no de patentes concedidas, los distintos procedimientos indican algo tan importante como las expectativas, lo que esperan tanto los investigadores como la institución, en este caso el CSIC, a la que están adscritos sobre ese trabajo.

Al igual que en el caso de la autoría y para ejemplificar este modelo o propuesta de estudio se han agrupado, en el Cuadro 15 , las solicitudes de patentes de cuatro centros del CSIC en función de los distintos procedimientos elegidos para su puesta en el mercado. Las entradas que se han establecido son:

- España (OEPM): agrupa las solicitudes de patentes que cada centro quiere proteger en el mercado español.

- $\quad$ España (OEPM) + PCT: agrupa las solicitudes de patentes que se optan al sistema PCT para ver las posibilidades que tienen en el mercado internacional.

- $\quad$ España $($ OEPM) + PCT +P. Nacionales: agrupa las solicitudes de patentes que una vez puestas en el mercado internacional han dado un tercer paso y han optado a los procedimientos nacionales de otros países. Permite ver cuáles son los países donde se quiere proteger este conocimiento.

- Europa (OEP): agrupa las solicitudes de patentes presentadas en la oficina de patentes europea, por lo que sólo en principio optan por el mercado europeo.

- $\quad$ España (OEPM)+ PCT + Europa: esta entrada agrupa las solicitudes de patentes que una vez gestionadas por el sistema PCT han optado por el mercado europeo.

CuAdro 15. Procedimientos y solicitudes de patentes

\begin{tabular}{|l|l|c|}
\hline CBM & Procedimiento & Patentes \\
\hline & España (OEPM) & 24 \\
\hline & España (OEPM) + PCT & 13 \\
\hline & España (OEPM) + PCT +P. Nacionales & 5 \\
\hline CID & España (OEPM) & 19 \\
\hline & España (OEPM) + PCT & 1 \\
\hline & España (OEPM) + PCT + P. Nacionales & 3 \\
\hline & Europa (OEP) & 1 \\
\hline CIB & España (OEPM) & 28 \\
\hline & España (OEPM)+ PCT & 14 \\
\hline & España (OEPM)+ PCT + P. Nacionales & 3 \\
\hline & España (OEPM)+ PCT + Europa & 4 \\
\hline CNB & España (OEPM) & 31 \\
\hline & España (OEPM) + PCT & 14 \\
\hline & España (OEPM) + PCT + P. Nacionales & 5 \\
\hline & Europa (OEP) & 5 \\
\hline & & \\
\hline
\end{tabular}

El análisis detenido de estos datos va a permitir conocer mejor cómo viaja el conocimiento generado por estos centros del CSIC y también a qué países pretende llegar. Utilizando un instrumento tan gráfico como es un mapa, seria interesante que este estudio permitiera finalmente realizar un mapa del conocimiento científico y tecnológico que, unido a los datos de las autorias, reflejara donde se originan las corrientes y cuáles son las vías de penetración de dicho conocimiento.

\section{La explotación}

Ya se ha mencionado que la tercera de las cuestiones que ilustra el modelo de estudio que se quiere plantear es la uti- 
lización que se hace de ese conocimiento en los diferentes lugares donde se consigue proteger. Cuántas de estas solicitudes de patentes finalmente se consigue proteger; este es un dato relevante tanto para las empresas como para los centros de investigación, ya que todo conocimiento que no se protege difícilmente se podrá explotar. Cuántas solicitudes pasan a ser explotadas. En qué condiciones se produce esta explotación: lo hace una empresa en exclusiva o lo comparte más de una empresa. Qué papel juega en el proceso de explotación el grupo de investigación, y por tanto el centro, que gestó el conocimiento. Quiénes son los demandantes 0 explotadores de este conocimiento (empresas nacionales o extranjeras, empresas de nueva creación, otros centros de investigación). Los datos invitan a hacer estudios concretos de las denominadas spin-offs y start-ups, o empresas que han surgido en el seno de la propia investigación, creadas, en su mayor parte por científicos formados y soportados económicamente por el sistema público de investigación, con el apoyo de capitales-riesgo, capaces de compatibilizar la producción de bienes con la consecución de sus investigaciones.

Son muchas las preguntas que se plantean, quizá más que las que en un trabajo como este se pueden resolver. La propia inercia de la investigación permitirá, en próximos trabajos ir dando respuestas, y cómo no, abriendo nuevas cuestiones. Todo ello, en definitiva, permitirá conocer mejor cómo se produce el conocimiento, cómo se gestiona y desarrolla para finalmente ver en qué casos realmente se da el paso a la innovación.

\section{NOTAS}

1 La Colección Española de Cultivos Tipo (CECT) tiene su origen en 1960, año en que el profesor Julio Rodríguez Villanueva comenzó a recoger y mantener cepas microbianas. Desde 1960 a 1968 la colección estuvo depositada en dos centros del CSIC, el Instituto Jaime Ferrán de Microbiologia y en el Instituto de Biologia Celular. Con el traslado del profesor Rodríguez Villanueva a la Universidad de Salamanca, la CECT pasó a formar parte del Departamento de Microbiología de la Facultad de Biologia de dicha universidad, donde estuvo hasta 1974, fecha en que se produjo un nuevo traslado, esta vez al Departamento de Biología de la Universidad del Pais Vasco en Bilbao, donde el profesor Federico Uruburu pasó a convertirse en su nuevo encargado. En 1980, la CECT se trasladó de nuevo a Valencia, donde ha permanecido desde entonces.

2 Las tablas han sido realizadas a partir de los datos cedidos por la Oficina de Transferencia de Tecnología del CSIC. Agradecemos a su director José Luis de Miguel, a Domingo Represa y a Marcos Palomo, su diligente colaboración.
3 La Oficina de Transferencia de Tecnología del CSIC agrupa las solicitudes por áreas. Para facilitar la elaboración de las tablas se ha asignado un número a cada una de ellas: 1 Humanidades y Ciencias Sociales; 2 Biologia y Biomedicina; 3 Recursos Naturales;4 Ciencias Agrarias; 5 Ciencia y Tecnologías Físicas; 6 Ciencia y Tecnologia de Materiales; 7 Ciencia y Tecnologia de Alimentos; 8 Ciencia y Tecnologías Quimicas.

4 Una solicitud hecha en el año 2004 no tiene asignado número de área, razón por la cual no coincide la cifra de solicitudes totales por años (83) con la de solicitudes por años y áreas para 2004 (82).

5 El año 2004 se ha omitido en esta tabla pues las cifras, debido a los plazos que debe cumplir el procedimiento PCT, no sólo no son representativas sino que pueden conducir a errores de interpretación.

6 La distribución de centros por comunidades es la siguiente. Madrid 5, Cataluña 3, Comunidad Valenciana 2, Andalucía 2 y Castilla y León 1.

\section{BIBLIOGRAFÍA}

Albert, A. (en prensa): Generación y trasferencia de conocimiento cientifico en ciencias de la vida y desarrollo tecnológico. El caso español, en Muñoz et al.: La posibilidad de la construcción de un espacio común del conocimiento en la Unión Europea: Un enfoque al problema desde España, Madrid: Academia Europea de Ciencias y Artes España.

Albert, A. \& Plaza, L. (2004): Avance del estudio estratégico de la biotecnología en España: descripción e indicadores, Madrid: Genoma España.

Azagra C., Joaquín M., Fernández de Lucio, I. \& Gutiérrez Garcia, A. (2003): University patents: output and input indicators ... of what?, Research Evaluation 12, 1, 5-16.

Bercovitz, A., et al. (1985): Derecho de patentes: España y la Comunidad Económica Europea, Barcelona: Ariel.

Buesa, M. (2002): El sistema regional de innovación de la Comunidad de Madrid, Documento de Trabajo del Instituto de Análisis Económico y Financiero $n^{\circ} 30$, Madrid: Universidad Complutense de Madrid.

Buesa, M. (2003): Ciencia y tecnología en la España democrática: la formación de un sistema nacional de innovación, ICE, 25 años de Constitución española, no 811 , 235-272. 
Buesa, M. \& Moler, J. (1998): Patrones del cambio tecnológico y política industrial. Un estudio de las empresas innovadoras madrileñas, Madrid: Civitas.

Fundación COTEC (2004): Libro blanco de la innovación en la Comunidad de Madrid. Informes sobre el sistema español de innovación. Fundación COTEC para la innovación tecnológica, Madrid.

Lanjow, J.0. \& Schankerman, M. (2004): Patent quality and research productivity: measuring innovation with multiple indicators, The Economic Journal 114, 441-465.

López Garcia, S. (1997): El Patronato Juan de la Cierva (1939-1960) I parte: las instituciones precedentes, Arbor CLVII, 619, 201-238.

López García, S. (1998): El Patronato Juan de la Cierva II parte: la organización y la financiación, Arbor CLIX, 625,1-44.

López Garcia, S. (1999): El Patronato Juan de la Cierva III parte: la investigación científica y tecnológica, Arbor CLXII, 637, 1-32.

Metcalfe, J. (1995): The economic foundation of technology policy: equilibrium and evolutionary perspectives, en P. Stoneman (ed.): Handbook of Economics of Innovation an Technology Change, Oxford: Blackwell.

Muñoz, E., Santesmases, M.J., López Facal, J. \& Todt, 0. (eds.) (2004): ¿Es posible la construcción de un espacio común del conocimiento?, Madrid: Academia Europea de Ciencias y Artes.

Represa, D., Castro, E. \& Fernández de Lucio, I. (2003): Promoción de la protección de los resultados de investigación pública española desde el Plan Nacional de I+D: balance de 10 años. Fundación, Tecnología, Universidad y Empresa, Madrid: OEl, Temas de lberoamérica.

Sáiz González, J.P. (1995): Propiedad industrial y revolución liberal. Historia del sistema de patentes español (17591929), Madrid: OEPM.

Sáiz González, J.P. (1996): Legis/ación histórica sobre propiedad industrial. España 1759-1929, Madrid: OEPM.

Sáiz González, J.P. (1999): Invención, patentes e innovación en la España contemporánea, Madrid: OEPM.
Santesmases, M.J. \& Muñoz, E. (1997): Establecimiento de la bioquímica y de la biología molecular en España. Madrid: Fundación Ramón Areces-CSIC.

Sanz Menéndez, L. \& Arias, E. (1998): Especialización y capacidades tecnológicas de las regiones españolas: un análisis a través de las patentes europeas, Economia Industrial 324 (VI), 105-122.

\section{LEGISLACIÓN}

Estatuto de la Propiedad Industrial. Real Decreto-Ley de 26 de julio de 1929.

Ley de Patentes. Ley 11/1986 de 20 de marzo. Reglamento de ejecución. Real Decreto $2245 / 1986$ de 10 de octubre.

Procedimiento general de concesión. Real Decreto 2245/1986 de 10 de octubre.

Procedimiento de concesión con examen previo. Real Decreto 812/2000 de 19 de mayo y Real decreto 996/2001 de 10 de septiembre.

Convenio Patente Europea firmado en Munich el 5 de octubre de 1973. BOE de 24-IX-1986.

Reglamento de ejecución del Convenio de Patente Europea. BOE 30-IX-1986.

Modificación de la Ley de Patentes, en todo lo referido a materia biológica, que recoge el Tratado de Budapest. Ley 10/2002 en vigor desde el 1-V-2002. 